\title{
Regulation of Spontaneous Contractions in Intact Rat Bladder Strips and the Effects of Hydrogen Peroxide
}

\author{
Mingshuai Wang, ${ }^{1,2}$ Nianzeng Xing, ${ }^{2}$ Liyang Wu, ${ }^{1,2}$ Wen-Chu Huang, ${ }^{1,3}$ \\ Zhenqun $\mathrm{Xu},{ }^{1,4}$ and Guiming Liu $\mathbb{1}^{1}$ \\ ${ }^{1}$ Department of Surgery, MetroHealth Medical Center, Case Western Reserve University, Cleveland, OH, USA \\ ${ }^{2}$ Department of Urology, Beijing Chao-Yang Hospital, Capital Medical University, Beijing, China \\ ${ }^{3}$ Division of Urogynecology, Department of Obstetrics and Gynecology, Mackay Memorial Hospital, Taipei, Taiwan \\ ${ }^{4}$ Department of Urology, Shengjing Hospital, China Medical University, Shenyang, China \\ Correspondence should be addressed to Guiming Liu; guiming.liu@case.edu
}

Received 23 August 2017; Revised 1 December 2017; Accepted 31 December 2017; Published 4 February 2018

Academic Editor: Kota V. Ramana

Copyright (c) 2018 Mingshuai Wang et al. This is an open access article distributed under the Creative Commons Attribution License, which permits unrestricted use, distribution, and reproduction in any medium, provided the original work is properly cited.

\begin{abstract}
Enhanced spontaneous contractions are associated with overactive bladder. Elevated levels of reactive oxygen species might contribute to enhanced spontaneous contractions. We investigated the regulation of spontaneous contractions and the effects of hydrogen peroxide $\left(\mathrm{H}_{2} \mathrm{O}_{2}\right)$ in intact rat bladder strips. The spontaneous contractions were measured using a tissue bath system. The vehicle or the specific activators/blockers were applied and followed by the application of $0.003 \mathrm{~g} \% \mathrm{H}_{2} \mathrm{O}_{2}$. The basal tension, amplitude, and frequency of spontaneous contractions were quantified. Nisoldipine and bisindolylmaleimide 1 had no effects on spontaneous contractions. SKF96365 and Y27632 decreased basal tension and amplitude. Ryanodine slightly increased frequency. Both iberiotoxin and NS-1619 increased amplitude. Apamin reduced frequency but increased amplitude. NS-309 inhibited both the amplitude and frequency. The basal tension and amplitude increased when $\mathrm{H}_{2} \mathrm{O}_{2}$ was applied. Pretreatment with NS-309 inhibited $\mathrm{H}_{2} \mathrm{O}_{2}$-elicited augmented amplitude and frequency, while pretreatment with Y-27632 inhibited the augmented basal tension. The combined application of NS-309 and Y27632 almost eliminated spontaneous contractions and its augmentation induced by $\mathrm{H}_{2} \mathrm{O}_{2}$. In conclusion, $\mathrm{Ca}^{2+}$ influx, Rho kinase activation, and SK channel inactivation play important roles in spontaneous contractions in intact bladder strips, whereas only latter two mechanisms may be involved in $\mathrm{H}_{2} \mathrm{O}_{2}$-elicited increased spontaneous contractions.
\end{abstract}

\section{Introduction}

The urinary bladder has two important functions, which are to store and expel urine. The detrusor smooth muscle of the bladder exhibits spontaneous contractile activity during the filling phase and in isolated detrusor strips $[1,2]$. The spontaneous activity cannot be abolished by tetrodotoxin, atropine, phentolamine, propranolol, or hexamethonium, indicating that the activity is not dependent on innervation $[1,2]$. The role of spontaneous activity may be to facilitate adjustment of muscle bundle lengths during bladder filling $[3,4]$. Under certain pathological conditions, the spontaneous activity could spread and initiate synchronous contractions throughout the detrusor, generating involuntary contractions $[5,6]$. Previous studies using denuded detrusor muscle strips suggested that calcium channels [7], largeconductance calcium-activated potassium [BK] channel [8], small-conductance calcium-activated potassium [SK] channel [9], Rho-associated coiled-coil kinase (ROCK) [10], and protein kinase $\mathrm{C}$ (PKC) [11] contributed to the regulation of smooth muscle spontaneous contractions.

Overactive bladder $(\mathrm{OAB})$ is prevalent in patients or animal models with diabetes [12], bladder outlet obstruction [13], chronic ischemia [14], ischemia/reperfusion [15], or ageing [16]. These pathophysiological conditions are also characterized by excessive accumulation of reactive oxygen species (ROS). Therefore, the elevated levels of ROS have been proposed to contribute to $\mathrm{OAB}[17,18]$. More directly, we generated an inducible, smooth muscle-specific manganese superoxide dismutase (MnSOD) gene knockout 
mouse model recently [19]. We found the depletion of MnSOD caused oxidative stress in the bladder and the mice presented bladder overactivity [19]. Higher levels of spontaneous activity were found in detrusor muscle strips from humans and animals with $\mathrm{OAB}[5,6]$. Rabbits with chronic moderate bladder ischemia presented bladder overactivity, along with increased levels of oxidative stress markers and spontaneous bladder contractions [14]. These studies indicated the enhanced spontaneous myogenic activity might be due to higher levels of ROS.

ROS include free radicals such as superoxide anion $\left(\mathrm{O}_{2}{ }^{--}\right)$, hydroxyl radical $\left({ }^{\circ} \mathrm{OH}\right)$, and nitric oxide (NO), and nonradical molecules like peroxynitrite (ONOO-) and hydrogen peroxide $\left(\mathrm{H}_{2} \mathrm{O}_{2}\right)$ [20, 21]. ROS have long been believed to play important roles in pathological conditions. However, recent evidence has shown that ROS may also function as a second messenger in a signaling cascade induced by changes in the ion channel activity in response to neurotransmitters and hormones $[22,23] . \mathrm{H}_{2} \mathrm{O}_{2}$ is an important naturally occurring ROS and has been used to investigate the effects of ROS [24, 25]. Masuda et al. showed that intravesical instillation of $\mathrm{H}_{2} \mathrm{O}_{2}(0.003-0.3 \mathrm{~g} \%)$ can induce bladder overactivity, including both a decrease in the intercontraction interval and an increase in the amplitude [18]. In addition, low concentrations of $\mathrm{H}_{2} \mathrm{O}_{2}$ have been shown to increase the contractile responses of the bladder detrusor muscle strips in a dose-dependent manner [26]. However, the effects of $\mathrm{H}_{2} \mathrm{O}_{2}$ on spontaneous contractions in intact bladder strips and the mechanisms involved have not been well investigated. The mucosa, including the urothelium, basement membrane, and lamina propria, can regulate detrusor contractions [27, 28]. In the present study, we investigated mechanisms of the regulation of spontaneous contractions and the effects of $\mathrm{H}_{2} \mathrm{O}_{2}$ in intact rat bladder strips.

\section{Materials and Methods}

2.1. Animals. Male Sprague-Dawley rats (10-12 weeks old, Harlan Laboratories, Indianapolis, IN) were used for this study. The animals were maintained with free access to laboratory chow and tap water in an animal facility under controlled temperature $\left(22^{\circ} \mathrm{C}\right)$, humidity $(55 \% \pm 10 \%)$, and lighting (12/12 $\mathrm{h}$ artificial light/dark cycle). The bladders were removed under $2 \%$ isoflurane anesthesia, and then animals were euthanized by a single intraperitoneal injection of pentobarbital $(200 \mathrm{mg} / \mathrm{kg})$. All protocols were approved by the Institutional Animal Care and Use Committee of Case Western Reserve University.

\subsection{Preparation of Rat Bladder Strips and Isometric Tension} Measurement. Urinary bladders were placed in cold oxygenated Krebs solution $\left(\mathrm{mM}: \mathrm{NaCl} 133, \mathrm{KCl} 4.7, \mathrm{NaHCO}_{3}\right.$ 16.3, $\mathrm{NaH}_{2} \mathrm{PO}_{4}$ 1.35, $\mathrm{MgSO}_{4} \cdot 7 \mathrm{H}_{2} \mathrm{O}$ 0.6, $\mathrm{CaCl}_{2} \cdot 2 \mathrm{H}_{2} \mathrm{O}$ 2.5, Dextrose $7.8, \mathrm{pH}=7.4)[29,30]$. After the surrounding adipose and connective tissues were removed, the trigone and dome area of bladders were cut and the bladder body was opened with a longitudinal incision. Full-thickness strips $(8-10 \mathrm{mg}$, $2 \mathrm{~mm}$ in width $\times 10 \mathrm{~mm}$ in length) were prepared as we described previously $[29,30]$. The strips were then mounted in a $20 \mathrm{ml}$ tissue bath (Radnoti, Radnoti Glass Technology INC, Monrovia, CA) containing $20 \mathrm{ml}$ Krebs solution aerated with $95 \% \mathrm{O}_{2}, 5 \% \mathrm{CO}_{2}$ to obtain a pH of 7.4 at $37^{\circ} \mathrm{C}$. One end of each strip was connected to a stationary glass hook, while the other end was attached to a force transducer (MLT0402, ADInstruments, Colorado Springs, CO, USA). Isometric contraction was recorded at a sampling rate of $20 \mathrm{~Hz}$ using a computerized data acquisition program (PowerLab 8/30 data acquisition system, ADInstruments, Colorado Springs, CO, USA) and stored on the computer for later analysis. After equilibration for 15 minutes at slack length, the strips were stretched with $1.0 \mathrm{gm}$ of tension and equilibrated for another 45 minutes. During the equilibration period, the bathing solution was changed every 15 minutes and strips were slightly stretched as needed to induce the maximal spontaneous rhythmic contraction. To exclude the possible neurogenic contribution of nerves contained in the bladder strips, all experiments were performed in the presence of a neuronal inhibitory cocktail including tetrodotoxin $(1 \mu \mathrm{M}$, a neuronal $\mathrm{Na}^{+}$channel blocker) and neurotransmitter receptor antagonists atropine $(1 \mu \mathrm{M}$, a muscarinic antagonist), phentolamine $(1 \mu \mathrm{M}$, an $\alpha$-adrenergic antagonist), propranolol $(1 \mu \mathrm{M}$, a $\beta$-adrenergic antagonist), and suramin $(10 \mu \mathrm{M}$, a purinergic antagonist) [31,32]. The strips, which developed less than 0.1 grams of spontaneous contractions amplitude, were excluded from further measurements.

2.3. Experimental Protocol and Data Analysis. After bladder strips were equilibrated, one of following test agents (specific activator or blocker) or vehicle (drug solvent) was introduced to the $20 \mathrm{ml}$ bath solution and incubated for 15 minutes: nisoldipine $\left(100 \mathrm{nM}\right.$, an L-type voltage-gated $\mathrm{Ca}^{2+}$ channel blocker), SKF96365 (10 $\mu \mathrm{M}$, inhibit store-operated $\mathrm{Ca}^{2+}$ channel, receptor-operated $\mathrm{Ca}^{2+}$ channel, and voltageoperated $\mathrm{Ca}^{2+}$ channel), ryanodine $\left(10 \mu \mathrm{M}\right.$, inhibits $\mathrm{Ca}^{2+}$ release from sarcoplasmic reticulum), iberiotoxin $(100 \mathrm{nM}$, a BK channel blocker), NS-1619 (30 $\mu \mathrm{M}$, a BK channel activator), apamin (100 nM, a SK channel blocker), NS-309 (10 $\mu \mathrm{M}$, a SK channel activator), bisindolylmaleimide 1 (BIS1, $2 \mu \mathrm{M}$, a protein kinase C inhibitor), or Y27632 $(10 \mu \mathrm{M}$, a ROCK inhibitor). The concentrations of the specific blockers or activators were chosen based on the previous studies [31-33]. Then, $20 \mu \mathrm{l}$ of $3 \mathrm{~g} \%$ (grams $/ 100 \mathrm{ml}$ ) $\mathrm{H}_{2} \mathrm{O}_{2}$ was added to the bath solution (the final concentration of $\mathrm{H}_{2} \mathrm{O}_{2}$ was $0.003 \mathrm{~g} \%$ ) and incubated for $15 \mathrm{~min}$. The concentration of $\mathrm{H}_{2} \mathrm{O}_{2}$ was chosen based on the previous in vivo [18] and in vitro $[25,26,34]$ studies. In addition, our own pilot experiment showed $0.003 \mathrm{~g} \% \mathrm{H}_{2} \mathrm{O}_{2}$ can induce obvious augmentation of spontaneous contractions. Data were collected using a PowerLab 8/30 data acquisition system and LabChart 6 Pro (Version 6.1.1) software (ADInstruments, Colorado Springs, CO, USA).

The timeline of the experimental protocol can be seen in Figure 1, which shows representative tracings of spontaneous contractions in bladder strips. For analysis, the tracings were divided into three sections according to the time of application of the different agents: Phase 1 is the period after application of the neuronal inhibitory cocktail only, Phase 2 is the period of incubation of the bladder strip with a test 


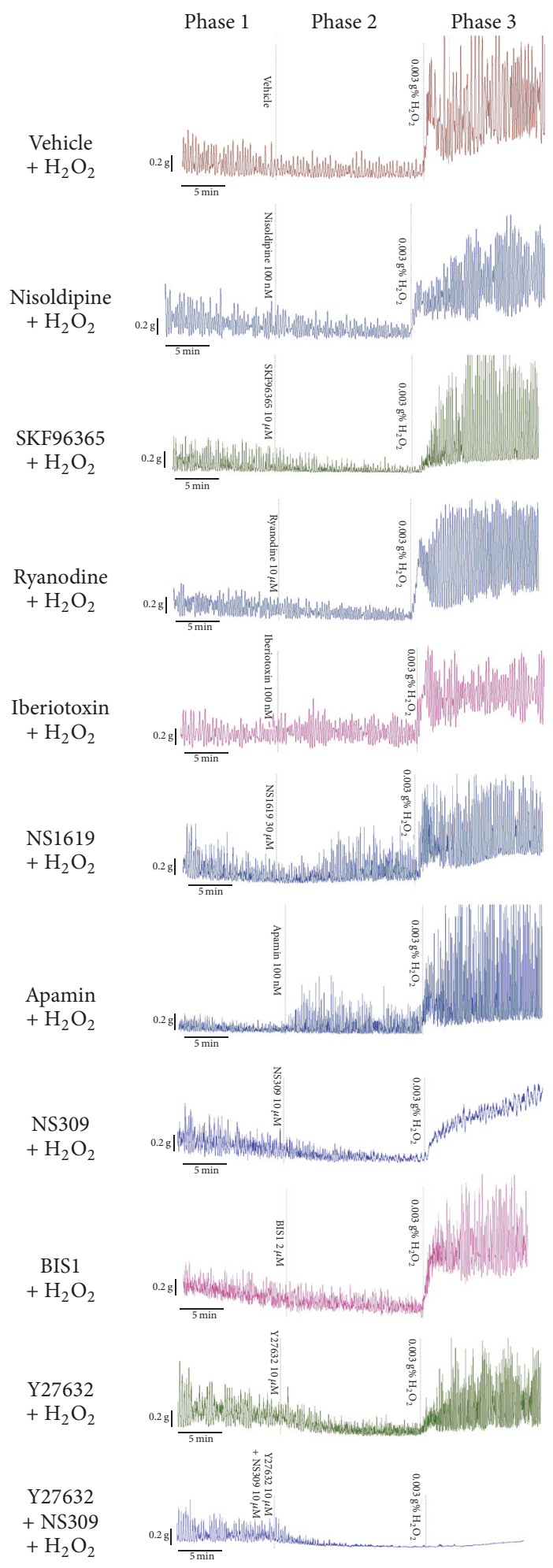

FIGURE 1: Representative tracings of spontaneous contractions of intact rat bladder strips before (Phase 1) and during (Phase 2) treatment with vehicle (0.1\% DMSO) or test agent [nisoldipine (100 nM), SKF96365 $(10 \mu \mathrm{M})$, ryanodine $(10 \mu \mathrm{M})$, iberiotoxin $(100 \mathrm{nM})$, NS1619 $(30 \mu \mathrm{M})$, apamin $(100 \mathrm{nM}), \mathrm{NS} 309(10 \mu \mathrm{M}), \mathrm{Y} 27632(10 \mu \mathrm{M})$, BIS-1 $(2 \mu \mathrm{M})$, and Y27632 + NS309 (each $10 \mu \mathrm{M})]$ and then incubated with $0.003 \mathrm{~g} \% \mathrm{H}_{2} \mathrm{O}_{2}$ (Phase 3). 




FIGURE 2: A zoomed image of spontaneous contraction tracings illustrating the quantified parameters. The spontaneous contractile events, the amplitude of which exceed $30 \%$ of the maximum response for the final $5 \mathrm{~min}$ in each $15 \mathrm{~min}$ incubation period, were used for analysis. For quantification of frequency, the contraction with multiple peaks due to partial relaxation (shown as a dashed arrow) was counted as one contraction event.

agent or vehicle, and Phase 3 is the period after addition of $\mathrm{H}_{2} \mathrm{O}_{2}$ to the bath containing inhibitory cocktail and test agent or vehicle. The responses during the final $5 \mathrm{~min}$ of each $15 \mathrm{~min}$ incubation period in different phases were used for analysis. Three parameters of spontaneous contractions, basal tension (the average of the lowest tension of phasic spontaneous contractions), amplitude (the average amplitude of spontaneous contractions), and frequency (number of contractions per minute), were measured using LabChart 6 Pro software. Figure 2 illustrated how the parameters were measured. The spontaneous contractile events, the amplitude of which exceed $30 \%$ of the maximum response for the final $5 \mathrm{~min}$ in each $15 \mathrm{~min}$ incubation period, were used for analysis as previously described [32]. In addition, the contraction with multiple peaks due to partially relaxation was counted as one contraction event.

The effects of a test agent on spontaneous contractions were determined by calculating the percentages of Phase 2 values to Phase 1 values when the test agent was applied in Phase 2 and comparing those percentages to the corresponding percentages when Phase 2 incubation was with vehicle alone. Similarly, the effects of a test agent on $\mathrm{H}_{2} \mathrm{O}_{2}$ induced altered spontaneous contractions were determined by comparing the percentages of Phase 3 values to Phase 1 values when the test agent was present in Phase 2 to the corresponding percentages in the absence of the test agent.

2.4. Drugs and Solutions. Tetrodotoxin, phentolamine, iberiotoxin, NS-1619, Y-27632, apamin, NS-309, and ryanodine were purchased from Abcam (Cambridge, MA, USA). Atropine, propranolol, suramin, nisoldipine, and SKF-96365 were purchased from Sigma-Aldrich (St. Louis, MO USA). Bisindolylmaleimide 1 and $\mathrm{H}_{2} \mathrm{O}_{2}$ were obtained from Cayman Chemical (Ann Arbor, MI, USA) and Fisher Scientific (Pittsburg, PA, USA), respectively.

NS-1619, nisoldipine, NS 309, and SKF-96365 were dissolved in dimethylsulphoxide (DMSO). Ryanodine was dissolved in $100 \%$ ethanol. All other drugs were prepared with water. Final ethanol and DMSO concentrations in the bath solution did not exceed $0.1 \%$ and were shown not to affect spontaneous contractions or bladder function in previous reports [35-37].

2.5. Statistical Analysis. Statistical analysis was performed using GraphPad Prism 6 (GraphPad Software, La Jolla, CA). The data are presented as mean \pm SEM. For comparison between vehicle and drug application group, unpaired nonparametric Mann-Whitney test was used. A $p$ value less than 0.05 was considered statistically significant.

\section{Results}

3.1. Rhythmic Spontaneous Contractions in Intact Bladder Strips Were Observed. As shown in Figure 1, rhythmic spontaneous contractions occurred in intact bladder strips in the presence of the neuronal inhibitory cocktail, suggesting a myogenic basis of the contraction. However, the basal tension and the contraction amplitude decreased within the investigated period. In the control (vehicle-treated) group (the data from water, ethanol, and DMSO-treated strips were pooled since less than $0.1 \%$ ethanol or DMSO did not affect spontaneous contractions in previous reports [35-37] and in our pilot study), the basal tension and amplitude decreased $13.6 \%$ and $32.5 \%$, respectively, from Phase 1 to Phase 2, while the frequency did not change significantly (Figure 1, Table 1).

3.2. Roles of Calcium Channels on Spontaneous Contractions in Intact Bladder Strips. Nisoldipine had no significant effect on spontaneous contractions of intact bladder strips (Figure 1, Table 1), whereas SKF96365 decreased the amplitude dramatically and basal tension slightly (Figure 1, Table 1). Application of ryanodine resulted in an increase in the frequency, but no significant change in the basal tension and amplitude (Figure 1, Table 1).

3.3. Roles of Potassium Channels on Spontaneous Contractions in Intact Bladder Strips. Iberiotoxin increased the basal tension and the amplitude of spontaneous contractions slightly, but did not affect frequency (Figure 1, Table 1). Interestingly, NS-1619 increased contraction amplitude, as well as basal tension significantly (Figure 1, Table 1). Apamin reduced frequency and increased amplitude dramatically but did not affect basal tension (Figure 1, Table 1). NS-309 markedly inhibited both the amplitude and frequency of spontaneous contractions but caused very little change in basal tension (Figure 1, Table 1).

3.4. Roles of $\mathrm{Ca}^{2+}$ Sensitivity-Related Protein Kinases on Spontaneous Contractions in Intact Bladder Strips. To study the roles of $\mathrm{Ca}^{2+}$ sensitivity-related protein kinases ROCK, and $\mathrm{PKC}$ in spontaneous contractions in bladder strips, PKC inhibitor bisindolylmaleimide I (BIS1) and the ROCK inhibitor Y27632 were used. As shown in Figure 1 and Table 1, BIS1 $(2 \mu \mathrm{M})$ did not affect the basal tension, amplitude, or frequency of spontaneous contractions in intact bladder strips. Y27632 $(10 \mu \mathrm{M})$ significantly inhibited the basal tension and 
TABLE 1: Effects of specific activators and blockers on spontaneous contractions in intact bladder strips (Phase 2 values as percentages of Phase 1 values).

\begin{tabular}{lcccc}
\hline Drugs (concentration) & $n$ & Basal tension (\%) & Amplitude $(\%)$ & Frequency $(\%)$ \\
\hline Control $\left(\right.$ vehicle $\left.+\mathrm{H}_{2} \mathrm{O}_{2}\right)$ & 13 & $86.4 \pm 2.36$ & $67.5 \pm 5.09$ & $103.4 \pm 3.29$ \\
Nisoldipine $(100 \mathrm{nM})$ & 6 & $79.5 \pm 4.86$ & $67.8 \pm 7.73$ & $103.3 \pm 3.41$ \\
SKF96365 $(10 \mu \mathrm{M})$ & 6 & $75.4 \pm 3.93^{*}$ & $30.7 \pm 4.48^{*}$ & $100.7 \pm 7.31$ \\
Ryanodine $(10 \mu \mathrm{M})$ & 5 & $89.8 \pm 8.20$ & $82.4 \pm 8.89$ & $124.8 \pm 8.57^{*}$ \\
Iberiotoxin $(100 \mathrm{nM})$ & 8 & $96.8 \pm 3.99^{*}$ & $92.4 \pm 4.31^{*}$ & $106.8 \pm 4.43$ \\
NS1619 $(30 \mu \mathrm{M})$ & 5 & $101.8 \pm 6.07^{*}$ & $152.4 \pm 14.19^{*}$ & $109.2 \pm 5.97$ \\
Apamin $(100 \mathrm{nM})$ & 9 & $84.7 \pm 5.64$ & $325.3 \pm 73.87^{*}$ & $82.2 \pm 5.32^{*}$ \\
NS309 $(10 \mu \mathrm{M})$ & 6 & $80.1 \pm 2.20$ & $61.4 \pm 6.44^{*}$ & $61.1 \pm 6.36^{*}$ \\
BIS1 $(2 \mu \mathrm{M})$ & 7 & $81.0 \pm 2.45$ & $68.78 \pm 4.97$ & $118.4 \pm 16.58$ \\
Y27632 $(10 \mu \mathrm{M})$ & 9 & $59.9 \pm 4.71^{*}$ & $45.7 \pm 2.02^{*}$ & $131.6 \pm 7.53^{*}$ \\
Y27632 $(10 \mu \mathrm{M})+\mathrm{NS309}(10 \mu \mathrm{M})$ & 5 & $50.9 \pm 1.19^{*}$ & $3.3 \pm 3.34^{*}$ & $14.7 \pm 14.71^{*}$ \\
\hline
\end{tabular}

Values are expressed as mean plus or minus SEM of 5 to 13 individual strips; ${ }^{*}$ significantly different from corresponding value in vehicle treated group $(p<$ $0.05)$.

amplitude of the contractions, whereas the frequency was increased.

3.5. Effects of Combined Application of ROCK Inhibitor and SK Channels Activator on Spontaneous Contractions in Intact Bladder Strips. Based on our observation that NS309 can inhibit the amplitude and frequency of spontaneous contractions and Y27632 can inhibit the basal tension and amplitude of the contractions, we expected that the combined application of NS-309 and Y27632 would eliminate the spontaneous contractions. Indeed, the combined application of NS-309 and Y27632 eliminated spontaneous contractions almost completely (Figure 1, Table 1).

3.6. $\mathrm{H}_{2} \mathrm{O}_{2}$ Induced Augmentation of Spontaneous Contractions in Intact Bladder Strips. The basal tension and amplitude of spontaneous contractions increased significantly in the vehicle-pretreated group when $0.003 \% \mathrm{H}_{2} \mathrm{O}_{2}$ was applied. Basal tension increased to $204.6 \%$ and amplitude increased to $216.4 \%$ of Phase 1 , whereas frequency was not affected (Figure 1 and Table 2).

3.7. Roles of Calcium Channels in the $\mathrm{H}_{2} \mathrm{O}_{2}$-Induced Augmentation of Spontaneous Contractions in Intact Bladder Strips. As shown in Figure 1 and Table 2, pretreatment with nisoldipine $(100 \mathrm{nM})$, SKF-96365 $(10 \mu \mathrm{M})$, or ryanodine $(10 \mu \mathrm{M})$ did not prevent the increases in basal tension and amplitude of spontaneous contractions induced by $0.003 \mathrm{~g} \%$ $\mathrm{H}_{2} \mathrm{O}_{2}$ and did not affect the frequency significantly compared to the vehicle-treated group.

3.8. Roles of Potassium Channels in the $\mathrm{H}_{2} \mathrm{O}_{2}$-Induced Augmentation of Spontaneous Contractions in Intact Bladder Strips. Neither the BK channel blocker iberiotoxin (100 nM) nor the BK channel activator NS-1619 $(30 \mu \mathrm{M})$ affected the $\mathrm{H}_{2} \mathrm{O}_{2}$-induced augmentation of spontaneous contractions (Figure 1, Table 2). Pretreatment with the SK channel blocker apamin $(100 \mathrm{nM})$ did not affect the $\mathrm{H}_{2} \mathrm{O}_{2}$-induced increase of the basal tension; however, it dramatically enhanced the effect of $\mathrm{H}_{2} \mathrm{O}_{2}$ on the amplitude (Figure 1, Table 2). The SK channel activator NS-309 $(10 \mu \mathrm{M})$ significantly prevented the increase in the amplitude of spontaneous contractions induced by $\mathrm{H}_{2} \mathrm{O}_{2}$ and caused a dramatic reduction in frequency. However NS-309 did not affect the $\mathrm{H}_{2} \mathrm{O}_{2}$-induced increase of the basal tension.

3.9. Roles of $\mathrm{Ca}^{2+}$ Sensitivity-Related Protein Kinases on the $\mathrm{H}_{2} \mathrm{O}_{2}$-Induced Augmentation of Spontaneous Contractions in Intact Bladder Strips. As illustrated in Figure 1, the PKC inhibitor BIS1 $(2 \mu \mathrm{M})$ had no effect on the $\mathrm{H}_{2} \mathrm{O}_{2}$-induced augmentation of spontaneous contractions. The ROCK inhibitor Y-27632 $(10 \mu \mathrm{M})$ markedly prevented the $\mathrm{H}_{2} \mathrm{O}_{2}$-elicited augmentation of basal tension and increased frequency slightly. However it did not attenuate the augmented amplitude induced by $\mathrm{H}_{2} \mathrm{O}_{2}$ (Figure 1, Table 2).

3.10. Effects of Combined Application of ROCK Inhibitor and SK Channels Activator on the $\mathrm{H}_{2} \mathrm{O}_{2}$-Induced Augmentation of Spontaneous Contractions in Intact Bladder Strips. Upon observing that NS-309 can decrease the amplitude and frequency of the $\mathrm{H}_{2} \mathrm{O}_{2}$-induced augmentation of spontaneous contractions and Y27632 can inhibit the basal tension of the augmented contractions, we postulated that the combined application of NS-309 and Y27632 would affect the $\mathrm{H}_{2} \mathrm{O}_{2}$ induced augmented spontaneous contractions. Indeed, the combined application of NS-309 and Y27632 significantly prevented the $\mathrm{H}_{2} \mathrm{O}_{2}$-induced augmentation of spontaneous contractions, particularly the amplitude (Figure 1, Table 2).

\section{Discussion}

In order to closely reflect the in vivo situation, we chose to use intact full-thickness bladder strips in this study instead of detrusor muscle layer only, which were used in most previous studies $[32,38]$. The spontaneous contractions may increase [39] or decrease [27] in intact bladder strips compared to the bladder smooth muscle only strips. Current studies demonstrated that rhythmic spontaneous contractions occurred in intact bladder strips and persisted in the presence of a neuronal inhibitory cocktail. The frequency of spontaneous 
TABLE 2: Effects of specific activators and blockers on $\mathrm{H}_{2} \mathrm{O}_{2}$-induced augmentation of spontaneous contractions in intact bladder strips (Phase 3 values as percentages of Phase 1 values).

\begin{tabular}{|c|c|c|c|c|}
\hline Drugs & $n$ & Basal tension (\%) & Amplitude (\%) & Frequency $(\%)$ \\
\hline Control (vehicle $+\mathrm{H}_{2} \mathrm{O}_{2}$ ) & 13 & $204.6 \pm 10.79$ & $216.4 \pm 22.75$ & $100.7 \pm 3.00$ \\
\hline Nisoldipine $+\mathrm{H}_{2} \mathrm{O}_{2}$ & 6 & $195.0 \pm 14.81$ & $236.9 \pm 47.62$ & $105.8 \pm 5.25$ \\
\hline SKF96365 + $\mathrm{H}_{2} \mathrm{O}_{2}$ & 6 & $174.2 \pm 8.85$ & $246.4 \pm 56.27$ & $101.5 \pm 4.95$ \\
\hline Ryanodine $+\mathrm{H}_{2} \mathrm{O}_{2}$ & 5 & $209.0 \pm 20.37$ & $253.8 \pm 52.82$ & $130.4 \pm 13.43$ \\
\hline Iberiotoxin $+\mathrm{H}_{2} \mathrm{O}_{2}$ & 8 & $238.8 \pm 13.63$ & $339.3 \pm 57.82$ & $96.6 \pm 8.39$ \\
\hline $\mathrm{NS} 1619+\mathrm{H}_{2} \mathrm{O}_{2}$ & 5 & $248.3 \pm 16.42$ & $318.1 \pm 55.97$ & $98.8 \pm 7.57$ \\
\hline Apamin $+\mathrm{H}_{2} \mathrm{O}_{2}$ & 9 & $222.5 \pm 13.48$ & $1025.7 \pm 217.71^{*}$ & $84.2 \pm 7.39$ \\
\hline $\mathrm{NS} 309+\mathrm{H}_{2} \mathrm{O}_{2}$ & 6 & $208.5 \pm 25.43$ & $84.49 \pm 13.55^{*}$ & $41.2 \pm 5.46^{*}$ \\
\hline $\mathrm{BIS} 1+\mathrm{H}_{2} \mathrm{O}_{2}$ & 7 & $211.0 \pm 22.01$ & $230.0 \pm 31.13$ & $104.9 \pm 9.13$ \\
\hline $\mathrm{Y} 27632+\mathrm{H}_{2} \mathrm{O}_{2}$ & 9 & $128.7 \pm 17.98^{*}$ & $195.4 \pm 35.64$ & $125.3 \pm 8.51^{*}$ \\
\hline $\mathrm{Y} 27632+\mathrm{NS} 309+\mathrm{H}_{2} \mathrm{O}_{2}$ & 5 & $97.4 \pm 4.72^{*}$ & $9.4 \pm 3.97^{*}$ & $31.6 \pm 19.23^{*}$ \\
\hline
\end{tabular}

Values are expressed as mean plus or minus SEM of 5 to 13 individual strips; ${ }^{*}$ significantly different from corresponding value in vehicle treated group $(p<$ $0.05)$.

contractions did not change. The basal tension and the amplitude decreased slightly over the investigated period, which is probably due to stress relaxation.

It is well established that contraction of smooth muscle such as detrusor is modulated primarily by the intracellular $\mathrm{Ca}^{2+}$ concentration [40]. Calcium influx and calcium release from sarcoplasmic reticulum have been indicated to play important roles in regulating smooth muscle contraction [40]. In the present study, SKF96365, which inhibits the calcium entry through store-operated $\mathrm{Ca}^{2+}$ channel, receptoroperated $\mathrm{Ca}^{2+}$ channel, and voltage-operated $\mathrm{Ca}^{2+}$ channel [41], can significantly inhibit spontaneous activity in intact bladder strips, indicating $\mathrm{Ca}^{2+}$ influx is involved. However, Ltype voltage-gated $\mathrm{Ca}^{2+}$ channel blocker nisoldipine, which has been shown to inhibit spontaneous contractions in bladder detrusor muscle $[27,32]$, did not affect spontaneous activity in intact bladder strips. In addition, the ryanodine $(10 \mu \mathrm{M})$, which was shown inhibiting ryanodine receptormediated $\mathrm{Ca}^{2+}$ release and suppressing spontaneous activity of the bladder detrusor muscle $[27,33]$, slightly increases the frequency of intact bladder strips. Therefore, current results in intact bladder strips are not fully consistent with those previously reported in detrusor muscle only [27]. It looks that mucosa, which consists of the urothelium, basement membrane, and lamina propria [28], may play an role in regulation of the spontaneous contractions. Mucosa may weaken or offset the effects of nisoldipine or ryanodine on spontaneous contractions and therefore maintain the amplitude in intact bladder strips. Urothelial cells express a variety of receptors and ion channels and can release diffusible agents, for example, prostaglandins, which could enhance spontaneous activity in the intact bladder $[28,42,43]$. The different results also could be due to the different species used. Guinea-pig and pig detrusor strips were used in the above-mentioned studies [27, 32, 33]. Another possible reason is that we only examined the effects of a single dose of nisoldipine (100 nM) [27] and ryanodine $(10 \mu \mathrm{M})$ on spontaneous contractions. The effects of different doses need to be examined in the future. Different doses of activators/blockers may have different effects on $\mathrm{Ca}^{2+}$ influx and release. Ryanodine has been reported to promote $\mathrm{Ca}^{2+}$ release at low concentrations, while locking up the ryanodine sensitive channels and reducing or preventing $\mathrm{Ca}^{2+}$ release at high concentrations [44].

Although all three subtypes of calcium-activated potassium channels $\left(\mathrm{K}_{(\mathrm{Ca})}\right)$ including large, intermediate, and small-conductance $\mathrm{K}_{(\mathrm{Ca})}(\mathrm{BK}, \mathrm{IK}$, and $\mathrm{SK}$ ) are present in the bladder, previous study suggested that IK is not important in regulation of bladder contraction [9], whereas the opening of BK and SK channels has been shown to cause detrusor smooth muscle relaxation in human [45] and rat [46] through the leak of potassium ions along their concentration gradient into the extracellular space. The current study showed that blocking BK channel with iberiotoxin slightly increased contraction amplitude of the intact bladder strip, which is the same as the results from the detrusor smooth muscle only strip. Surprisingly, BK channel activator NS1619 $(30 \mu \mathrm{M})$ also increases the basal tension and amplitude in intact bladder strips. This is contrary to the previous studies performed in bladder detrusor muscle strips [47, 48]. The phenomenon may be due to the presence of mucosa. Although BK channels are mainly located in detrusor smooth muscle cells, several studies showed that mucosa also express BK channels [49, 50]. The function of $\mathrm{BK}$ channels in urothelium is not clear. Blocking SK channels with apamin reduced contraction frequency and increased the contraction amplitude, while SK channel opener NS309 decreased the spontaneous contractions amplitude and frequency significantly in intact bladder strips. These results are consistent with the studies performed in detrusor muscle strips $[31,38]$.

In addition to intracellular $\mathrm{Ca}^{2+}$ concentration, $\mathrm{Ca}^{2+}$ sensitization also plays an important role in smooth muscle contraction [40]. ROCK and PKC are two major molecules that regulate $\mathrm{Ca}^{2+}$ sensitization [51]. ROCK inhibitor Y27632 decreased both the amplitude and basal tension but increased the frequency of spontaneous contractions in intact bladder strips. This result indicated that ROCK activation plays an 
important role in spontaneous contractions, whereas PKC inhibitor BIS1 did not suppress the spontaneous contractile amplitude, frequency, and basal tension, suggesting that PKC pathway may not play an important role in mediating spontaneous contractions in intact bladder strips. The data support a previous study [10] which showed that the ROCK inhibitors $(1 \mu \mathrm{M}$ and $3 \mu \mathrm{M}$ Y-27632 and $10 \mu \mathrm{M}$ HA-1077) reduced both basal myosin light chain phosphorylation and tone in rabbit detrusor at the optimum length for muscle contraction, whereas the PKC inhibitor $(1 \mu \mathrm{M}$ GF109203X) did not produce a significant reduction in myosin light chain phosphorylation and tone. However, the results are not consistent with another study which showed PKC inhibitor helped to maintain the amplitude of spontaneous contractions of rabbit detrusor smooth muscle strips [11]. In addition to inhibiting the amplitude of spontaneous contractions, NS309 inhibits the frequency, whereas Y27632 suppresses the base tension. Therefore, we expected that when NS309 and Y27632 were used together, the overall inhibitory response would be enhanced. We found indeed that the application of NS309 and Y27632 together almost eliminated the spontaneous contractions in intact bladder strips.

A previous study showed that $\mathrm{H}_{2} \mathrm{O}_{2}$ can induce contraction in rat detrusor smooth muscle strips [26]. Our interest was to determine the effects of $\mathrm{H}_{2} \mathrm{O}_{2}$ on the spontaneous contractions and the underlying mechanisms in intact bladder strips. We found $\mathrm{H}_{2} \mathrm{O}_{2}$ can increase the basal tension and amplitude, but not the frequency of spontaneous contractile activity in intact bladder strips. The increased amplitude may be due to direct effects or indirect effects through increasing basal detrusor smooth muscle tone. Pretreatment with nisoldipine, SKF96365, and ryanodine did not prevent $\mathrm{H}_{2} \mathrm{O}_{2}$-induced augmentation of the basal tension and amplitude of spontaneous contractions, which suggests $\mathrm{Ca}^{2+}$ influx and ryanodine receptor-related $\mathrm{Ca}^{2+}$ release from sarcoplasmic reticulum are not essential to the $\mathrm{H}_{2} \mathrm{O}_{2}$-induced increase of spontaneous contractions in intact bladder strips. In addition, pretreatment with BK channel activator NS-1619 or blocker iberiotoxin and PKC inhibitor Bis1 did not affect $\mathrm{H}_{2} \mathrm{O}_{2}$-elicited increases of the basal tension and amplitude. However, SK channel activator NS-309 pretreatment significantly inhibited the $\mathrm{H}_{2} \mathrm{O}_{2}$-elicited increase of amplitude, and SK channel blocker apamin pretreatment enhanced the $\mathrm{H}_{2} \mathrm{O}_{2}$-elicited increase of the amplitude. These results indicated that $\mathrm{H}_{2} \mathrm{O}_{2}$ increased the amplitude of spontaneous contractions at least partially through the deactivation of SK channel. $\mathrm{H}_{2} \mathrm{O}_{2}$ regulates the activity of the proteins either through direct oxidative modification or indirectly through modification of associated signaling molecules [52]. Wei et al. used the patch-clamp technique to study the effect of $\mathrm{H}_{2} \mathrm{O}_{2}$ on SK channel in the cortical collecting duct. They found the addition of $\mathrm{H}_{2} \mathrm{O}_{2}$ decreased the activity of the SK channel by stimulating protein tyrosine kinase, P38, and ERK in the cortical collecting duct and then enhancing the internalization of the SK channel [53]. In addition, ROCK inhibitor Y-27632 significantly inhibited the $\mathrm{H}_{2} \mathrm{O}_{2}$ elicited increase of basal tension, suggesting that $\mathrm{H}_{2} \mathrm{O}_{2}$ may increase $\mathrm{Ca}^{2+}$ sensitivity by regulation of the ROCK pathway.
Multiple studies have demonstrated that $\mathrm{H}_{2} \mathrm{O}_{2}$ can enhance the activation of ROCK and mediate vascular contraction $[54,55]$. In spontaneously hypertensive rats, increased ROCK activation was involved in the $\mathrm{H}_{2} \mathrm{O}_{2}$-induced contraction of mesenteric resistance arteries [55]. $\mathrm{H}_{2} \mathrm{O}_{2}$ activates ROCK possibly through the activation of the upstream molecule, RhoA, but not direct activation of ROCK, since ROCK is not directly redox sensitive [56]. Considering the different effects of NS309 and Y27632 on the $\mathrm{H}_{2} \mathrm{O}_{2}$-induced augmentation of spontaneous contractions, we postulated that they might have synergistic effects when they were applied together. The results actually showed that combined application of Y27632 and NS-309 significantly prevented the $\mathrm{H}_{2} \mathrm{O}_{2}$-induced increases of basal tension, amplitude, and frequency of the spontaneous contractions.

\section{Conclusions}

Our results suggest that $\mathrm{Ca}^{2+}$ influx, Rho kinase activation, and SK channel deactivation may play important roles in the spontaneous contractions in intact bladder strips, whereas only latter two mechanisms may be involved in $\mathrm{H}_{2} \mathrm{O}_{2}$ induced augmentation of spontaneous contractions. Previous studies indicated that excessive accumulation of ROS [17$19,57]$ and enhanced spontaneous contractions $[5,6,14]$ are related to $\mathrm{OAB}$, indicating high levels of ROS may contribute to the enhanced spontaneous contractions. The current study explored the potential mechanisms of the $\mathrm{H}_{2} \mathrm{O}_{2}$-induced augmentation of spontaneous contractions through pharmacological approach. One limitation is that we used just a single dose of activator/blocker based on the previous reports. Dose-response effects of the specific activators/blockers on $\mathrm{H}_{2} \mathrm{O}_{2}$ induced augmentation of spontaneous contractions need to be investigated. In addition, future experiments are warranted to (1) confirm the current results using electrophysiological techniques; (2) determine the effects of combined application of SK channel activator and ROCK inhibitor on ROS-related OAB in animal models.

\section{Conflicts of Interest}

The authors declare that they have no conflicts of interest.

\section{Authors' Contributions}

G. Liu and M. Wang conceptualized the study. M. Wang, L. $\mathrm{Wu}$, and W. Huang performed experiments. N. Xing participated in the experimental design and partially supported $M$. Wang. M. Wang, Z. Xu, and G. Liu analyzed and interpreted the data. M. Wang and G. Liu wrote the paper.

\section{Acknowledgments}

This study was partially supported by the National Institutes of Health National Institute of Diabetes and Digestive and Kidney Diseases (Grant 1R01 DK110567). Mingshuai Wang was supported by the Physician Study Abroad Scholarship Program from Beijing (2009-2-02), China. 


\section{References}

[1] R. M. Levin, M. R. Ruggieri, S. Velagapudi, D. Gordon, B. Altman, and Wein. A. J., "Relevance of spontaneous activity to urinary bladder function: an in vitro and in vivo study," The Journal of Urology, vol. 136, pp. 517-521, 1986.

[2] A. F. Brading, "A myogenic basis for the overactive bladder," Urology, vol. 50, pp. 57-67, 1997.

[3] A. F. Brading, "Spontaneous activity of lower urinary tract smooth muscles: correlation between ion channels and tissue function," The Journal of Physiology, vol. 570, no. 1, pp. 13-22, 2006.

[4] J. E. Speich, C. W. Wilson, A. M. Almasri, J. B. Southern, A. P. Klausner, and P. H. Ratz, "Carbachol-induced volume adaptation in mouse bladder and length adaptation via rhythmic contraction in rabbit detrusor," Annals of Biomedical Engineering, vol. 40, no. 10, pp. 2266-2276, 2012.

[5] C. H. Fry, G.-P. Sui, N. J. Severs, and C. Wu, "Spontaneous activity and electrical coupling in human detrusor smooth muscle: Implications for detrusor overactivity?" Urology, vol. 63, no. 3, pp. 3-10, 2004.

[6] K.-E. Andersson, "Detrusor myocyte activity and afferent signaling," Neurourology and Urodynamics, vol. 29, no. 1, pp. 97106, 2010.

[7] H. Hashitani, A. F. Brading, and H. Suzuki, "Correlation between spontaneous electrical, calcium and mechanical activity in detrusor smooth muscle of the guinea-pig bladder," British Journal of Pharmacology, vol. 141, no. 1, pp. 183-193, 2004.

[8] G. V. Petkov, "Central role of the BK channel in urinary bladder smooth muscle physiology and pathophysiology," American Journal of Physiology-Regulatory, Integrative and Comparative Physiology, vol. 307, no. 6, pp. R571-R584, 2014.

[9] S. A. Y. Afeli, E. S. Rovner, and G. V. Petkov, "SK but not IK channels regulate human detrusor smooth muscle spontaneous and nerve-evoked contractions," American Journal of PhysiologyRenal Physiology, vol. 303, no. 4, pp. F559-F568, 2012.

[10] P. H. Ratz and A. S. Miner, "Length-dependent regulation of basal myosin phosphorylation and force in detrusor smooth muscle," American Journal of Physiology-Regulatory, Integrative and Comparative Physiology, vol. 284, no. 4, pp. R1063-R1070, 2003.

[11] J. A. Hypolite, Q. Lei, S. Chang et al., "Spontaneous and evoked contractions are regulated by $\mathrm{PKC}$-mediated signaling in detrusor smooth muscle: Involvement of BK channels," American Journal of Physiology-Renal Physiology, vol. 304, no. 5, pp. F451-F462, 2013.

[12] N. Xiao, Z. Wang, Y. Huang, F. Daneshgari, and G. Liu, "Roles of polyuria and hyperglycemia in bladder dysfunction in diabetes," The Journal of Urology, vol. 189, no. 3, pp. 1130-1136, 2013.

[13] C. M. Callaghan, C. Schuler, R. E. Leggett, and R. M. Levin, "Effect of severity and duration of bladder outlet obstruction on catalase and superoxide dismutase activity," International Journal of Urology, vol. 20, no. 11, pp. 1130-1135, 2013.

[14] Q. Zhang, M. Siroky, J.-H. Yang, Z. Zhao, and K. Azadzoi, "Effects of ischemia and oxidative stress on bladder purinoceptors expression," Urology, vol. 84, no. 5, pp. 1249.el-1249.e7, 2014.

[15] J.-H. Shin, G.-H. Kim, K.-H. Song, Y.-G. Na, C.-K. Sul, and J.S. Lim, "Protective effect of $\mathrm{N}$-acetylcysteine against ischemia/ reperfusion injury in rat urinary bladders," Cell Biochemistry \& Function, vol. 32, no. 1, pp. 24-30, 2014.

[16] P. J. Gomez-Pinilla, M. F. Gomez, K. Swärd et al., "Melatonin restores impaired contractility in aged guinea pig urinary bladder," Journal of Pineal Research, vol. 44, no. 4, pp. 416-425, 2008.

[17] Y.-B. Huang, M.-W. Lin, Y. Chao, C.-T. Huang, Y.-H. Tsai, and P.C. Wu, "Anti-oxidant activity and attenuation of bladder hyperactivity by the flavonoid compound kaempferol," International Journal of Urology, vol. 21, no. 1, pp. 94-98, 2014.

[18] H. Masuda, K. Kihara, K. Saito et al., "Reactive oxygen species mediate detrusor overactivity via sensitization of afferent pathway in the bladder of anaesthetized rats," BJU International, vol. 101, no. 6, pp. 775-780, 2008.

[19] G. Liu, R. A. Elrashidy, N. Xiao et al., "Bladder function in mice with inducible smooth muscle-specific deletion of the manganese superoxide dismutase gene," American Journal of Physiology-Cell Physiology, vol. 309, no. 3, pp. C169-C178, 2015.

[20] V. I. Lushchak, "Free radicals, reactive oxygen species, oxidative stress and its classification," Chemico-Biological Interactions, vol. 224, pp. 164-175, 2014.

[21] P. D. Ray, B. Huang, and Y. Tsuji, "Reactive oxygen species (ROS) homeostasis and redox regulation in cellular signaling," Cellular Signalling, vol. 24, no. 5, pp. 981-990, 2012.

[22] K. Kiselyov and S. Muallem, "ROS and intracellular ion channels," Cell Calcium, vol. 60, no. 2, pp. 108-114, 2016.

[23] K. M. Holmström and T. Finkel, "Cellular mechanisms and physiological consequences of redox-dependent signalling," Nature Reviews Molecular Cell Biology, vol. 15, no. 6, pp. 411-421, 2014.

[24] L. Malone, C. Schuler, RE. Leggett, and R. M. Levin, "The effect of in vitro oxidative stress on the female rabbit bladder contractile response and antioxidant levels," ISRN Urology, vol. 2013, Article ID 639685, 6 pages, 2013.

[25] K. Kojima, H. Kume, S. Ito et al., "Direct effects of hydrogen peroxide on airway smooth muscle tone: Roles of Ca2+ influx and Rho-kinase," European Journal of Pharmacology, vol. 556, no. 1-3, pp. 151-156, 2007.

[26] J. H. Han, M. Y. Lee, S. Y. Lee et al., "Effect of low concentrations of hydrogen peroxide on the contractile responses of rat detrusor smooth muscle strips," European Journal of Pharmacology, vol. 638, no. 1-3, pp. 115-120, 2010.

[27] S. A. Buckner, I. Milicic, A. V. Daza, M. J. Coghlan, and M. Gopalakrishnan, "Spontaneous phasic activity of the pig urinary bladder smooth muscle: Characteristics and sensitivity to potassium channel modulators," British Journal of Pharmacology, vol. 135, no. 3, pp. 639-648, 2002.

[28] L. A. Birder, M. Ruggieri, M. Takeda et al., "How does the urothelium affect bladder function in health and disease?: ICI-RS 2011," Neurourology and Urodynamics, vol. 31, no. 3, pp. 293-299, 2012.

[29] G. Liu and F. Daneshgari, "Alterations in neurogenically mediated contractile responses of urinary bladder in rats with diabetes," American Journal of Physiology-Renal Physiology, vol. 288, no. 6, pp. F1220-F1226, 2005.

[30] F. Daneshgari, G. Liu, and P. B. Imrey, “Time Dependent Changes in Diabetic Cystopathy in Rats Include Compensated and Decompensated Bladder Function," The Journal of Urology, vol. 176, no. 1, pp. 380-386, 2006.

[31] G. M. Herrera, T. J. Heppner, and M. T. Nelson, "Regulation of urinary bladder smooth muscle contractions by ryanodine receptors and BK and SK channels," American Journal of Physiology-Regulatory, Integrative and Comparative Physiology, vol. 279, no. 1, pp. R60-R68, 2000.

[32] T. Imai, T. Okamoto, Y. Yamamoto et al., "Effects of different types of $\mathrm{K}+$ channel modulators on the spontaneous myogenic 
contraction of guinea-pig urinary bladder smooth muscle," Acta Physiologica Scandinavica, vol. 173, no. 3, pp. 323-333, 2001.

[33] V. Y. Ganitkevich and G. Isenberg, "Contribution of $\mathrm{Ca}(2+)$-induced $\mathrm{Ca} 2+$ release to the $[\mathrm{Ca} 2+] \mathrm{i}$ transients in myocytes from guinea-pig urinary bladder." The Journal of Physio$\log y$, vol. 458, no. 1, pp. 119-137, 1992.

[34] K. Aikawa, R. E. Leggett, and R. M. Levin, "Effect of age on hydrogen peroxide mediated contraction damage in the male rat bladder," The Journal of Urology, vol. 170, no. 5, pp. 20822085, 2003.

[35] O. Z. Shenfeld, K. A. McCammon, P. F. Blackmore, and P. H. Ratz, "Rapid effects of estrogen and progesterone on tone and spontaneous rhythmic contractions of the rabbit bladder," Urolithiasis, vol. 27, no. 5, pp. 386-392, 1999.

[36] L. Merrill and M. A. Vizzard, "Intravesical TRPV4 blockade reduces repeated variate stress-induced bladder dysfunction by increasing bladder capacity and decreasing voiding frequency in male rats," American Journal of Physiology-Regulatory, Integrative and Comparative Physiology, vol. 307, no. 4, pp. R471R480, 2014.

[37] D. E. Artim, F. A. Kullmann, S. L. Daugherty, H.-Y. Wu, and W. C. De Groat, "Activation of the nitric oxide-cGMP pathway reduces phasic contractions in neonatal rat bladder strips via protein kinase G," American Journal of Physiology-Renal Physiology, vol. 297, no. 2, pp. F333-F340, 2009.

[38] S. P. Parajuli, K. L. Hristov, R. P. Soder, W. F. Kellett, and G. V. Petkov, "NS309 decreases rat detrusor smooth muscle membrane potential and phasic contractions by activating SK3 channels," British Journal of Pharmacology, vol. 168, no. 7, pp. 16111625, 2013.

[39] H. Akino, C. R. Chapple, N. McKay et al., "Spontaneous contractions of the pig urinary bladder: The effect of ATP-sensitive potassium channels and the role of the mucosa," BJU International, vol. 102, no. 9, pp. 1168-1174, 2008.

[40] A. P. Somlyo and A. V. Somlyo, "Signal transduction by G-proteins, Rho-kinase and protein phosphatase to smooth muscle and non-muscle myosin II," The Journal of Physiology, vol. 522, no. 2, pp. 177-185, 2000.

[41] A. Singh, M. E. Hildebrand, E. Garcia, and T. P. Snutch, "The transient receptor potential channel antagonist SKF96365 is a potent blocker of low-voltage-activated T-type calcium channels," British Journal of Pharmacology, vol. 160, no. 6, pp. 14641475, 2010.

[42] A. Kanai, J. Roppolo, Y. Ikeda et al., "Origin of spontaneous activity in neonatal and adult rat bladders and its enhancement by stretch and muscarinic agonists," American Journal of Physiology-Renal Physiology, vol. 292, no. 3, pp. F1065-F1072, 2007.

[43] M. H. Hawthorn, C. R. Chapple, M. Cock, and R. ChessWilliams, "Urothelium-derived inhibitory factor(s) influences on detrusor muscle contractility in vitro," British Journal of Pharmacology, vol. 129, no. 3, pp. 416-419, 2000.

[44] G. Meissner, "Ryanodine activation and inhibition of the Ca2+ release channel of sarcoplasmic reticulum," The Journal of Biological Chemistry, vol. 261, no. 14, pp. 6300-6306, 1986.

[45] S. Oger, D. Behr-Roussel, D. Gorny et al., "Effects of potassium channel modulators on myogenic spontaneous phasic contractile activity in human detrusor from neurogenic patients," $B J U$ International, vol. 108, no. 4, pp. 604-611, 2011.

[46] M. Kita, T. Yunoki, K. Takimoto et al., "Effects of bladder outlet obstruction on properties of $\mathrm{Ca} 2+$-activated $\mathrm{K}+$ channels in rat bladder," American Journal of Physiology-Regulatory, Integrative and Comparative Physiology, vol. 298, no. 5, pp. R1310-R1319, 2010.

[47] J. J. Layne, B. Nausch, S.-P. Olesen, and M. T. Nelson, "BK channel activation by NS11021 decreases excitability and contractility of urinary bladder smooth muscle," American Journal of Physiology-Regulatory, Integrative and Comparative Physiology, vol. 298, no. 2, pp. R378-R384, 2010.

[48] R. P. Soder and G. V. Petkov, "Large conductance Ca2+-activated K+ channel activation with NS1619 decreases myogenic and neurogenic contractions of rat detrusor smooth muscle," European Journal of Pharmacology, vol. 670, no. 1, pp. 252-259, 2011.

[49] M. Li, Y. Sun, J. M. Simard, J.-Y. Wang, and T. C. Chai, "Augmented bladder urothelial polyamine signaling and block of BK channel in the pathophysiology of overactive bladder syndrome," American Journal of Physiology-Cell Physiology, vol. 297, no. 6, pp. C1445-C1451, 2009.

[50] H. Zakoji, H. Kobayashi, M. Yoshiyama, M. Takeda, and I. Araki, "Expression of Large Conductance, Voltage- and $\mathrm{Ca}^{2+}$ Activated $\mathrm{K}^{+}$(BK) Channels in Human Urinary Bladder: Alteration of Subunit Expression Profile in Association with Bladder Outlet Obstruction," Open Journal of Urology, vol. 03, no. 02, pp. 47-52, 2013.

[51] J. Singh and S. Rattan, "Role of PKC and RhoA/ROCK pathways in the spontaneous phasic activity in the rectal smooth muscle," American Journal of Physiology-Gastrointestinal and Liver Physiology, vol. 304, no. 8, pp. G723-G731, 2013.

[52] G. A. Knock and J. P. T. Ward, "Redox regulation of protein kinases as a modulator of vascular function," Antioxidants \& Redox Signaling, vol. 15, no. 6, pp. 1531-1547, 2011.

[53] Y. Wei, Z. Wang, E. Babilonia, H. Sterling, P. Sun, and W. Wang, "Effect of hydrogen peroxide on ROMK channels in the cortical collecting duct," American Journal of Physiology-Renal Physiology, vol. 292, no. 4, pp. F1151-F1156, 2007.

[54] O. Al-Shboul and A. Mustafa, "Effect of oxidative stress on Rho kinase II and smooth muscle contraction in rat stomach," Canadian Journal of Physiology and Pharmacology, vol. 93, no. 6, pp. 405-411, 2015.

[55] A. B. García-Redondo, A. M. Briones, S. Martínez-Revelles et al., "C-Src, ERK1/2 and Rho kinasemediate hydrogen peroxideinduced vascular contraction in hypertension: Role ofTXA2, $\mathrm{NAD}(\mathrm{P}) \mathrm{H}$ oxidase andmitochondria," Journal of Hypertension, vol. 33, no. 1, pp. 77-87, 2015.

[56] L. Jin, Z. Ying, and R. C. Webb, "Activation of Rho/Rho kinase signaling pathway by reactive oxygen species in rat aorta," American Journal of Physiology-Heart and Circulatory Physiology, vol. 287, no. 4, pp. H1495-H1500, 2004.

[57] M. Nomiya, K. Sagawa, J. Yazaki et al., "Increased bladder activity is associated with elevated oxidative stress markers and proinflammatory cytokines in a rat model of atherosclerosisinduced chronic bladder ischemia," Neurourology and Urodynamics, vol. 31, no. 1, pp. 185-189, 2012. 


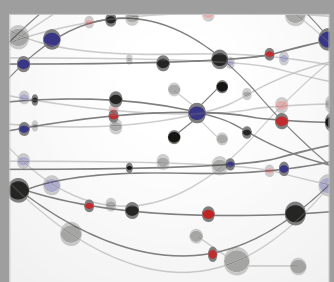

The Scientific World Journal
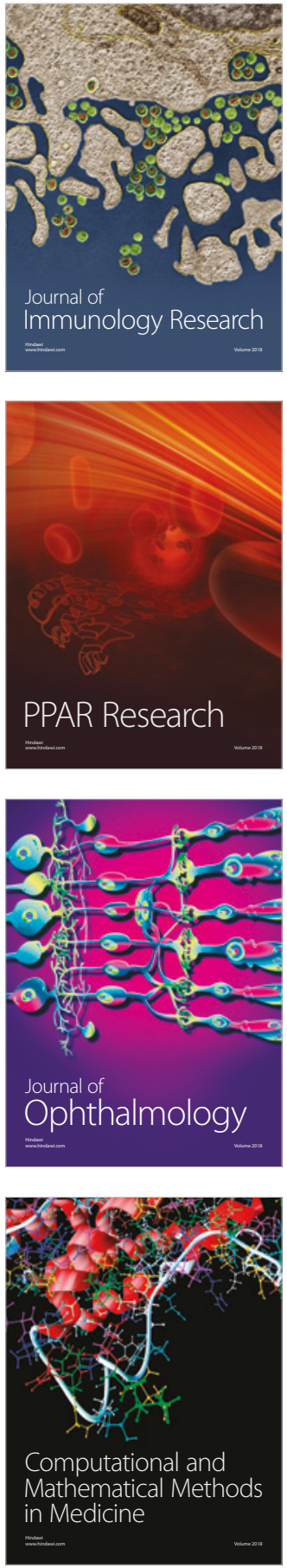

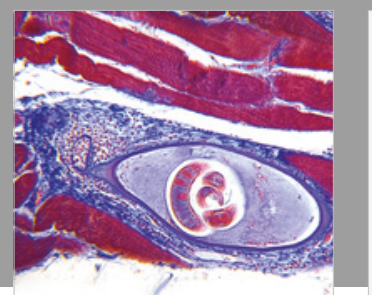

Gastroenterology Research and Practice

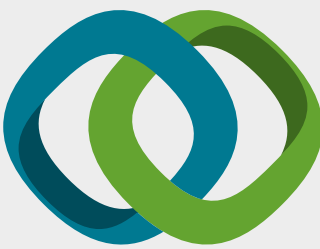

\section{Hindawi}

Submit your manuscripts at

www.hindawi.com


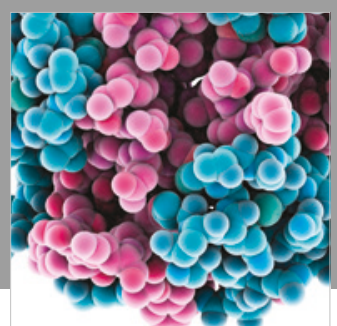

ournal of

Diabetes Research

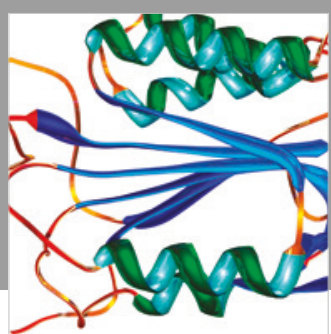

Disease Markers
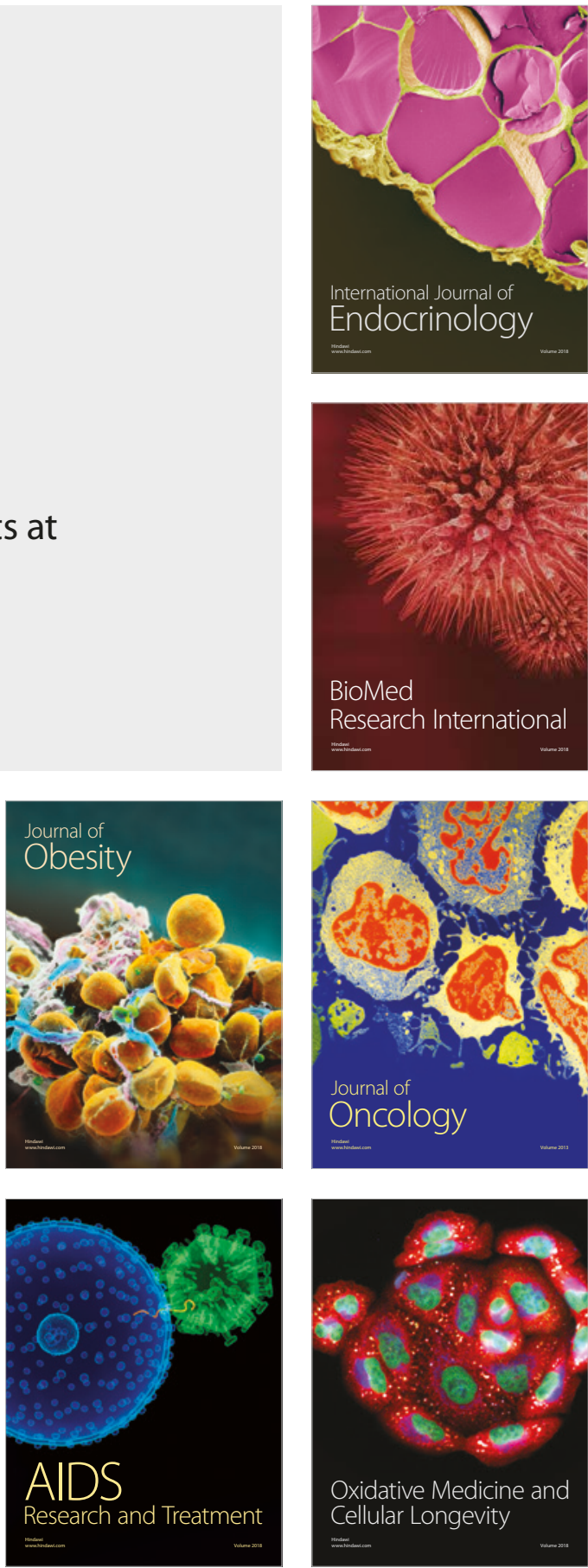